Was hielt das 20. Jahrhundert im Innersten zusammen? Wie lässt sich die deutsche Geschichte im „Zeitalter der Extreme“ deuten? Welche Leitbegriffe und Periodisierungen bieten sich an? Was ergibt sich daraus für Gesamtdarstellungen zur deutschen Zeitgeschichte? Ariane Leendertz stellt sich diesen Fragen. Sie setzt sich intensiv mit dem Konzept der Zeitbögen auseinander, das Anselm Doering-Manteuffel entwickelt und 2014 in den Vierteljahrsheften für Zeitgeschichte vorgestellt hat, unterzieht die Begrifflichkeit einer kritischen Prüfung und zeigt Stärken und Schwächen des Konzepts auf. Ausgehend von Doering-Manteuffels drittem Zeitbogen plädiert die Autorin dafür, die politische Ökonomie sowie die Ideen- und Politikgeschichte des Neoliberalismus stärker als bisher in der historischen Forschung zu berücksichtigen.

Ariane Leendertz

\title{
Zeitbögen, Neoliberalismus und das Ende des Westens, oder: Wie kann man die deutsche Geschichte des 20. Jahrhunderts schreiben?
}

\section{Synthesen}

Der amerikanische Historiker Charles S. Maier äußerte sich zur Jahrtausendwende in einem seitdem oft zitierten Aufsatz ausgesprochen skeptisch zum analytischen Wert einer Geschichte des 20. Jahrhunderts. Das 20. Jahrhundert stelle für die historische Analyse nur bedingt einen sinnvollen zeitlichen Rahmen dar, denn die Konzentration auf diese Zeitspanne erschwere die Sicht auf strukturelle Entwicklungen jenseits der Ereignisgeschichte und politischer Zäsuren. ${ }^{1}$ Maier benannte damit eine der Herausforderungen, mit denen Historikerinnen und Historiker konfrontiert sind, wenn sie sich an einer Gesamtdarstellung zur Geschichte Deutschlands im 20. Jahrhundert versuchen. Eine weitere Herausforderung ist es, sich mit dem auseinanderzusetzen, was Maier etwas despektierlich als „moralische Narrative“ bezeichnete. ${ }^{2}$ Etwas anders formuliert, ist die Geschichte des 20. Jahrhunderts nicht nur eine Domäne professioneller Historikerinnen und Historiker, sondern ebenfalls Gegenstand intellektueller Debatten, journalistischer und medialer Aufbereitung, öffentlicher Diskussionen um nationale Identität sowie (geschichts-)politischer Deutungen und Interpretationen. Die Geschichte des 20. Jahrhunderts ist mit dem kulturellen Gedächtnis und der öffentlichen

\footnotetext{
${ }^{1}$ Vgl. Charles S. Maier, Consigning the Twentieth Century to History. Alternative Narratives for the Modern Era, in: The American Historical Review 105 (2000), S. 807-831.

Für die Lektüre des vorliegenden Aufsatzes, Kommentare und Kritik danke ich Michael Hochgeschwender, Anette Schlimm, Martin Geyer, Silke Mende, Thomas Etzemüller und Elke Seefried.

${ }^{2}$ Ebenda, S. 807.
} 
Geschichte verwoben. Auch in Museen und Fernsehdokumentationen, in Spielfilmen und Romanen oder zu Jubiläen und Gedenktagen wird die Geschichte des vergangenen Jahrhunderts erzählt. Jenseits der Fachhistorie, so Maier, verkörpere das 20. Jahrhundert eine „moralische Epoche“, eine dunkle historische Periode, gekennzeichnet von einem erbarmungslosen Kampf der Ideologien, von Entmenschlichung und unermesslicher Gewalt gegenüber Zivilisten, von Massenmord und Genozid. Die internationale Fachwissenschaft stehe vor der Frage, wie sich diese „moralische Epoche“ zur strukturellen Periode institutionellen Wandels verhalte. ${ }^{3}$

Mit dem Spannungsfeld zwischen öffentlicher und fachwissenschaftlicher Geschichte sowie zwischen Ereignis-, Katastrophen- und Strukturgeschichte gehen Synthesen zur deutschen Geschichte des 20. Jahrhunderts unterschiedlich um. In der Historiografie ist dabei immer noch der Umgang mit der Sonderwegsthese umstritten. Heinrich August Winkler konstruierte seine Darstellung selbstbewusst als deutsche Nationalgeschichte, hingeschrieben auf den Fluchtpunkt der Wiedervereinigung im Jahr 1990. Winkler zufolge war Deutschland erst jetzt im Westen angekommen und hatte damit seine drei Sonderwege beendet: den jahrhundertealten „antiwestlichen“, dessen Wurzeln Winkler im Spätmittelalter verortete, den „postnationalen“ der Bundesrepublik und den „internationalistischen“ der DDR. ${ }^{4}$ Hans-Ulrich Wehler hielt im fünften Band seiner Gesellschaftsgeschichte dagegen, dass Deutschland bis 1914 immer ein unbestrittener Teil der westlichen Welt gewesen und die Bundesrepublik schon nach 1945 in den westlichen Kulturkreis zurückgekehrt sei - anders als die „sowjetische Satrapie in Ostdeutschland“.

Sowohl Wehler als auch Winkler betteten ihre Geschichten des 20. Jahrhunderts in viel längere Zeiträume ein. Wehler setzte allerdings die bei ihm nachgeordneten politischen Zäsuren in Beziehung zu Kontinuitäten und Diskontinuitäten im Bereich der wirtschaftlichen Entwicklung, der politischen Ökonomie und der Herrschafts- und Sozialstruktur, während bei Winkler die politischen Zäsuren die Darstellung strukturieren und er sich vor allem für politische Geschichte, politische Kultur und Debatten über nationale Identität interessierte. Wehlers Unternehmen war überdies mit dem analytisch anspruchsvollen Programm der Gesellschaftsgeschichte verknüpft, die mit Hilfe der Modernisierungstheorie eine neue Sicht auf die deutsche Geschichte ermöglichen und die Spezifika der deutschen Entwicklung herausarbeiten sollte, um den Nationalsozialismus jenseits der klassischen politik- und ideologiegeschichtlichen Ansätze zu erklären.

Die eigene Zeitzeugenschaft verbindet Wehler (Jahrgang 1931) und Winkler (1938) nun auch mit Ulrich Herbert (1951) und Anselm Doering-Manteuffel

\footnotetext{
${ }^{3}$ Ebenda, S. 812. Mit Charles S. Maier, Once Within Borders. Territories of Power, Wealth, and Belonging since 1500, Cambridge/London 2016, liegt jetzt seine in der Frühen Neuzeit beginnende, um das Strukturprinzip der Territorialität zentrierte Langzeitstudie vor.

${ }^{4}$ Vgl. Heinrich August Winkler, Der lange Weg nach Westen, Bd. 2: Deutsche Geschichte vom „Dritten Reich“ bis zur Wiedervereinigung, München 2000, S. 655.

${ }^{5}$ Hans-Ulrich Wehler, Deutsche Gesellschaftsgeschichte. Bundesrepublik und DDR 19491990, München 2008, S.424f., Zitat S. 425. „Westlich“ hieß bei Wehler: liberal, demokratisch und modern im Sinne der Moderne.
} 
(1949), wobei Herberts und Doering-Manteuffels Lebensgeschichten ausschließlich mit der Bundesrepublik verflochten sind ${ }^{6}$ Ersterer hat seine Geschichte Deutschlands im 20. Jahrhundert bereits abgeschlossen, während letzterer in konzeptionellen Aufsätzen die Möglichkeiten durchspielt, die deutsche Geschichte jenseits der üblichen Zäsuren und Interpretationsansätze zu schreiben. Orientiert an dieser Leitfrage und im punktuellen Vergleich mit Herbert soll im Folgenden Doering-Manteuffels Konzept der Zeitbögen diskutiert werden. ${ }^{7}$ Im Mittelpunkt stehen dabei einige Kritikpunkte, die Peter Hoeres dazu 2015 ebenfalls in den Vierteljahrsheften für Zeitgeschichte (VfZ) geäußert hat. Dies betrifft zunächst den Vorwurf, Doering-Manteuffel schreibe in den Zeitbögen die Sonderwegsthese fort. Vor allem aber sieht Hoeres im Konzept der Westernisierung eine analytisch-normative Verengung angelegt, die teleologisch, anachronistisch und erkenntnishemmend sei, zu apodiktisch vorgetragenen Fehlurteilen führe und in eine whigistische Triumphgeschichte des Westens münde, die sich blind gegenüber einer Schadensbilanz der westlichen Modernisierung zeige. ${ }^{8}$

Diese Annahmen beruhen meines Erachtens auf einem Missverständnis, das sich mit Hilfe eines historiografiegeschichtlichen Rückgriffs aufklären lässt. Im Konzept der Westernisierung, das in den zeitgeschichtlichen Fachdebatten der $1990 \mathrm{er}$ Jahre verwurzelt ist, ist weder eine normative noch eine teleologische Verengung angelegt. Die Krux liegt vielmehr darin, wie Doering-Manteuffel im Entwurf der Zeitbögen den Begriff des Westens und den Begriff des Liberalen füllt. Seine Interpretation scheint einen normativen Gehalt zu haben, der vor allem in der Gleichsetzung von „liberal“ und „westlich“, im Konstrukt eines „angloatlantischen Modells“ und in der Diagnose eines „liberalen und sozialen Konsenses“ in der Bundesrepublik bis Anfang der 1970er Jahre zum Ausdruck kommt. ${ }^{9}$ Vor diesem Hintergrund kann die Geschichte der Zeit „nach dem Boom“ nur im Modus einer Verfallsgeschichte erzählt werden, was an Eric Hobsbawms Interpretation vom Ende des Golden Age der Nachkriegsjahrzehnte erinnert. ${ }^{10}$

Dieser Aufsatz wird sich somit auf den zweiten und dritten Zeitbogen, also auf die Zeit seit den 1930er Jahren konzentrieren. Mit Bezug auf die anhaltende Debatte zur Periode „nach dem Boom“ sollen darüber hinaus einige Forschungsperspektiven zur Rolle des Nationalstaats und zur Ideen- und Politikgeschichte des

\footnotetext{
${ }^{6}$ Vor einiger Zeit unternahm Paul Nolte, Die Historiker der Bundesrepublik. Rückblick auf eine „lange“ Generation, in: Merkur 53 (1999), S. 413-432, den Versuch, die Kohorten um Hans-Ulrich Wehler, Gerhard A. Ritter, Wolfgang J. Mommsen und Hans Mommsen generationell zu deuten, wobei sich letzten Endes die Ansichten über die auch in anderen Fächern, in Geistesleben und Politik einflussreichen „45er“ bestätigten. Die nachrückende Generation, gemeinhin als „68er“ tituliert, hielt er für „schwer zu identifizieren“.

${ }^{7}$ Vgl. Anselm Doering-Manteuffel, Die deutsche Geschichte in den Zeitbögen des 20. Jahrhunderts, in: Vierteljahrshefte für Zeitgeschichte 62 (2014), S. 321-348.

${ }^{8}$ Vgl. Peter Hoeres, Gefangen in der analytisch-normativen Westernisierung der Zeitgeschichte. Eine Kritik am Konzept der Zeitbögen, in: Vierteljahrshefte für Zeitgeschichte 63 (2015), S. 427-436.

${ }^{9}$ Vgl. dazu unten, S. 195 u. S. 203.

${ }^{10}$ Vgl. Eric Hobsbawm, Das Zeitalter der Extreme. Weltgeschichte des 20. Jahrhunderts, München 1995.
} 
Neoliberalismus skizziert werden, die Doering-Manteuffel vollkommen zu Recht ins Zentrum des dritten Zeitbogens stellt. Anders als Hoeres halte ich den Neoliberalismus für einen geeigneten Interpretationsrahmen, um Transformationen der nationalen und internationalen politischen Ökonomie sowie gesamtgesellschaftlichen Wandel zu untersuchen. Die gegenwartsnahe Zeitgeschichte vermag neue Sichtweisen auf das lange 20. Jahrhundert zu eröffnen und sollte sich dabei nicht scheuen, in historisch orientierten politikwissenschaftlichen und soziologischen Arbeiten Anregungen für Theorie und Empirie zu suchen.

\section{Zur Strukturierung der deutschen Geschichte in drei Zeitbögen}

Die drei Zeitbögen dienen Doering-Manteuffel dazu, die deutsche Geschichte jenseits der bekannten Zäsuren zu periodisieren und damit zu strukturieren. Die Periodisierung orientiert sich nicht an der gängigen Ereignisgeschichte, sondern differenziert zwischen drei „Konstellationen“. Die Zeitbögen kennzeichneten jeweils spezifische „[s]trukturelle Grundmuster und ideelle Ordnungsentwürfe, die das Handeln in Politik, Wirtschaft und Gesellschaft transnational formatier[t]en“. ${ }^{11}$ Damit ist jene Perspektive der „erweiterten“, „neuen“ oder gesellschaftlich eingebetteten Ideengeschichte umrissen, die Doering-Manteuffel seit den 1990er Jahren in Tübingen in zahlreichen Forschungsprojekten etabliert hat. ${ }^{12}$ Charakterisiert als „Gesellschaftsgeschichte handlungssteuernder Ideen“, liegt sein Augenmerk auf Vorstellungen und Ordnungskonzepten, die im sozialen und politischen Geschehen „formende Wirkung“ entfalteten. ${ }^{13}$ Ähnlich wie Herbert sucht Doering-Manteuffel im eingangs beschriebenen Spannungsfeld zwischen politischer Ereignis-, deutschlandspezifischer Katastrophen- und analytischer Strukturgeschichte einen Ansatz, der diese drei Dimensionen integrieren kann. Herbert wählte hierfür das Konzept der Hoch- beziehungsweise Industriemoderne, die für ihn die Zeit zwischen 1890 und 1980 prägte. Die politischen und gesellschaftlichen Ordnungsentwürfe, die aus den ideologischen Strömungen Liberalismus /liberale Demokratie, Bolschewismus / Kommunismus und extremer Nationalismus / Nationalsozialismus erwuchsen, interpretierte er als unterschiedliche Antworten auf die Herausforderungen der modernen Industriegesellschaft. ${ }^{14}$

Während Herberts Binnenperiodisierung klassisch politikgeschichtlich bleibt, löst Doering-Manteuffel sie vollständig auf. Der erste Zeitbogen reicht von den 1890er Jahren bis zum Zweiten Weltkrieg, der zweite von den 1930er Jahren bis in die 1970er Jahre, der dritte von den 1970er Jahren bis in die Gegenwart. Das ist neu, interessant und eine Herausforderung für die Darstellung, denn so wird erstens das chronologische Gerüst der Eckdaten eingeebnet; zweitens scheint es -

${ }^{11}$ Doering-Manteuffel, Deutsche Geschichte, S. 322.

${ }^{12}$ In diesem Kontext ist auch meine Dissertation zu verorten; vgl. Ariane Leendertz, Ordnung schaffen. Deutsche Raumplanung im 20. Jahrhundert, Göttingen 2008.

13 Doering-Manteuffel, Deutsche Geschichte, S. 324.

${ }^{14}$ Vgl. Ulrich Herbert, Geschichte Deutschlands im 20. Jahrhundert, München 2014, und ders., Europe in High Modernity. Reflections on a Theory of the 20th Century, in: Journal of Modern European History 5 (2007), S. 5-21. 
auf den ersten Blick - an einer durchgängigen master narrative zu fehlen, die bei Wehler die Modernisierung, bei Winkler die Ankunft im Westen und bei Herbert das challenge and response-Modell ist. Das Konzept der Zeitbögen ist nicht brandneu, sein Urheber hat es bereits 2009 in einem Aufsatz dargelegt, damals aber noch von „Zeitschichten“ gesprochen; ein Begriff, der von Reinhart Koselleck geprägt wurde und bildlich die zeitliche Überlappung quasi geologisch auf- und nebeneinander geschichteter Ordnungsentwürfe vermittelt, die parallel existierten oder mit der Zeit ins Sediment absanken. ${ }^{15}$ Die Periodisierung ist seitdem dieselbe geblieben, jedoch hat Doering-Manteuffel den interpretativen Akzent verschoben. 2009 sah er das gesellschaftlich prägende Ordnungsdenken in der Periode des ersten Bogens von einer Erschöpfung der liberalen Fortschrittsideen, einem Verbindlichkeitsverlust des bürgerlichen Liberalismus und einer antihistoristischen Negation geschichtlicher Entwicklung gekennzeichnet, der die totalitären Denksysteme gesetzte, „ewige“ Ordnungen entgegenstellten. ${ }^{16}$ In Bogen zwei dominierten Vorstellungen von Ordnung als Struktur, gesellschaftlicher Modernisierung und organisierten Fortschritts, manifestiert etwa im fordistischen Produktionsmodell, im Planungsdenken oder in der Figur des Experten, die systemübergreifend sowohl im NS-Deutschland, in der Sowjetunion, im demokratischen Amerika oder der Bundesrepublik und der DDR zur Entfaltung kamen. Für Bogen drei schließlich betonte Doering-Manteuffel eine Auflösung des Fortschrittsbegriffs und des Ordnungsdenkens in Strukturen zugunsten von Netzwerken sowie fluider Relationen.

Dieser Ansatz war hoch abstrakt und wesentlich stärker um die heuristische Kategorie des Ordnungsdenkens arrangiert als der neuere Entwurf von 2014, in dem Doering-Manteuffel seine Interpretation, bogenübergreifend, auf den Antagonismus Liberalismus - Antiliberalismus zuspitzt. Das ist die master narrative, die seine Interpretation der deutschen Geschichte in transnationaler Perspektive bestimmen soll: Der „Kampf um die Durchsetzung oder Überwindung des angloatlantischen“ beziehungsweise „westlich-liberalen Ordnungsmodells“, bündig mit „Marktwirtschaft und Demokratie“ beschrieben, bildet für ihn den Grundkonflikt der deutschen Geschichte zwischen dem Ende des Ersten Weltkriegs und der Wiedervereinigung. ${ }^{17}$ Das scheint tatsächlich sehr nach Winkler, sehr nach einer Ankunft im Westen, möglicherweise sogar nach einem deutschen Sonderweg zu klingen.

\footnotetext{
${ }^{15}$ Vgl. Anselm Doering-Manteuffel, Konturen von Ordnung in den Zeitschichten des 20. Jahrhunderts, in: Thomas Etzemüller (Hrsg.), Die Ordnung der Moderne. Social Engineering im 20. Jahrhundert, Bielefeld 2009, S. 41-64.

${ }^{16}$ Vgl. zu diesem Interpretament Anselm Doering-Manteuffel, Mensch, Maschine, Zeit. Fortschrittsbewußtsein und Kulturkritik im ersten Drittel des 20. Jahrhunderts, in: Jahrbuch des Historischen Kollegs 2003, S. 91-119.

${ }^{17}$ Doering-Manteuffel, Deutsche Geschichte, S. 324.
} 


\section{Deutscher Sonderweg, Westen und Westernisierung}

Ist in Doering-Manteuffels Zeitbögen und im Konzept der Westernisierung die von Winkler reaktivierte und noch gesteigerte Sonderwegsthese Bielefelder Prägung eingeschrieben, wie Hoeres meint? Mit dem Sonderweg und dem Westen sind zwei Kategorien genannt, die Debatten und Forschungsfragen in der deutschen Zeitgeschichte von den 1970er bis in die 1990er Jahre geprägt haben. Beide sind eng mit der Geschichte des Fachs und zugleich mit der politischen Geschichte der Bonner Republik verflochten. Beide Debatten kreisten den noch bis in die frühen 1960er Jahre als Betriebsunfall der deutschen Geschichte relativierten Nationalsozialismus von unterschiedlichen Seiten ein. Die Sonderwegsthese erwuchs aus der im Nachklang der Fischer-Kontroverse entwickelten Frage nach den spezifischen gesellschaftlichen Bedingungen und Dispositionen, die Deutschland - anders als andere demokratische Industrienationen, namentlich Großbritannien, Frankreich und die USA - in die NS-Herrschaft führten. Als Erklärung fungierte das Abweichen von einem Modernisierungspfad, für den mit wenig empirischem Material ein idealtypischer Normalweg postuliert wurde. Die Sonderwegsthese ist wissenschaftlich fundiert und differenziert kritisiert worden, diese Kritik dient aber gerne als Keule: Wer heute noch einen deutschen Sonderweg suggeriert, ist fachwissenschaftlich von vorgestern und hält politisch an einem moralischen Geschichtsnarrativ fest, das die deutsche Geschichte als Vor- und Nachgeschichte des Nationalsozialismus konzipiert. Doch es führt eben auch analytisch oder strukturgeschichtlich kein Weg daran vorbei: Die deutsche Entwicklung mündete im Holocaust, und jede Darstellung zur deutschen Geschichte im 20. Jahrhundert muss erklären, wie es dazu kam. Gegenüber deterministischen Pfadabhängigkeiten wird heute auf die Kombination verschiedener gesellschaftlicher und politischer Entwicklungen, Ereignisse, Ideen und Dispositionen sowie eigendynamischer Prozesse und Kontingenzen verwiesen. Dieses historisch spezifische Faktorenbündel kann man, wenn man so will, als deutschen Sonderweg bezeichnen.

Zur Sonderwegsthese hat sich Doering-Manteuffel in seiner Rezension von Winklers „Der lange Weg nach Westen“ kritisch positioniert, ihr aber Berechtigung zugesprochen mit Bezug auf die „Ideen von 1914“ und die darum gruppierte Geisteshaltung der wilhelminischen Eliten. ${ }^{18}$ Insofern liegt Hoeres richtig, wenn er im Zeitbögen-Konzept Reflexe der Sonderwegsthese bemerkt. Der deutschnationale Chauvinismus am Vorabend des Weltkriegs wäre in der Tat zu Entwicklungen in anderen europäischen Staaten in Beziehung zu setzen, um vor diesem Hintergrund die deutschen Spezifika der antiliberalen Orientierung herausarbeiten zu können. Wie ist es aber nun um die deutsche Teilhabe am Westen und die Westernisierung bestellt? Will Doering-Manteuffel letztlich nichts anderes als Herbert und Winkler, die Hoeres sämtlich „mit stabilem normativen Werkzeug

${ }^{18}$ Zit. nach Anselm Doering-Manteuffel, Eine politische Nationalgeschichte für die Berliner Republik. Überlegungen zu Heinrich August Winklers „Der lange Weg nach Westen“, in: Geschichte und Gesellschaft 27 (2001), S. 446-462, hier S. 448. 
ausgestattet“ sieht, ${ }^{19}$ nämlich die deutsche Geschichte teleologisch und normativ auf ihren Platz im Westen hinschreiben, und dies noch dazu ohne transnationale oder globale Perspektivierung? ${ }^{20}$

Blicken wir zunächst auf Doering-Manteuffels teils sehr subtile Kritik an Winkler, in der er zugleich die Kernfrage des Westernisierungsansatzes auf den Punkt brachte: „Wie [...] hat sich der Wandel von einer obrigkeitlichen, antiindividualistischen, antiliberal und antisemitisch disponierten Gesellschaft zu einer individualistisch, liberal und pluralistisch geprägten Gesellschaft von freien Bürgern in der parlamentarischen Demokratie vollzogen?"21 Das könne Winkler allein mit der politischen Einbindung in die NATO, der europäischen Integration sowie in seiner Konzentration auf politische Eliten und Geschichtsdeutungen nicht erklären. Genau diese Frage bildete seit den 1990er Jahren das Gravitationszentrum von Doering-Manteuffels Sicht auf die Geschichte der Bundesrepublik. Ging es in den Fachdebatten im Zusammenhang mit der Sonderwegsthese zwischen den 1960er und 1980er Jahren um die Vorgeschichte des Nationalsozialismus (und im Wesentlichen um das 19. Jahrhundert), so begann in den 1970er Jahren auch die konsequente Historisierung der Bundesrepublik, deren „Erfolgsgeschichte“ zu ihrem 25. Geburtstag 1974 fast ungläubiges Staunen auslöste. ${ }^{22}$ Im Konzept der Westernisierung kam eine neue Akzentsetzung zum Ausdruck, die in engem Bezug zu den in den 1990er Jahren boomenden Forschungen zur Amerikanisierung stand. Diese befassten sich in breiter Fächerung mit der Frage des US-amerikanischen Einflusses in der westdeutschen Wirtschaft, vor allem aber in der Konsum- und Populärkultur, ebenso mit deutschen Amerikabildern sowie mit transatlantischen Austauschbeziehungen, die in der Regel als Einbahnstraße verstanden wurden, von Amerika zur Alten Welt. ${ }^{23}$

Neben der politischen Westintegration und der kulturellen und wirtschaftlichen Amerikanisierung war, so Doering-Manteuffel 1995, nach der ideellen Westorientierung der Deutschen, dem sich wandelnden Verständnis beziehungsweise Selbstverständnis des Westens und den Zuschreibungen und Werthaltungen zu fragen, die im Nachkriegsdeutschland mit dem Westen assoziiert wurden. ${ }^{24}$ Diese Forschungsfrage ist natürlich vor dem politischen Hintergrund des Kalten Kriegs,

\footnotetext{
${ }^{19}$ Hoeres, Gefangen, S. 427. In einer recht polemischen Rezension zu Ulrich Herbert erweckt Hoeres den Eindruck, es gehe weniger um eine prinzipielle Kritik an normativen Orientierungen in der Geschichtsschreibung, sondern vielmehr um eine Kritik an normativen und politischen Orientierungen, die Hoeres nicht teilt; Peter Hoeres, Rezension zu Geschichte Deutschlands im 20. Jahrhundert, in: Jahrbuch Extremismus \& Demokratie 27 (2015), S. 331-334.

${ }^{20}$ Vgl. Hoeres, Gefangen, S. 432.

${ }^{21}$ Doering-Manteuffel, Politische Nationalgeschichte, S. $456 \mathrm{f}$.

${ }^{22}$ Vgl. Richard Löwenthal/Hans-Peter Schwarz (Hrsg.), Die zweite Republik. 25 Jahre Bundesrepublik. Eine Bilanz, Stuttgart 1974.

${ }^{23}$ Vgl. Philipp Gassert, Amerikanismus, Antiamerikanismus, Amerikanisierung. Neue Literatur zur Sozial-, Wirtschafts- und Kulturgeschichte des amerikanischen Einflusses in Deutschland und Europa, in: Archiv für Sozialgeschichte 39 (1999), S. 531-561.

${ }^{24}$ Vgl. Anselm Doering-Manteuffel, Dimensionen von Amerikanisierung in der deutschen Gesellschaft, in: Archiv für Sozialgeschichte 35 (1995), S. 1-34, hier S. 25 f.
} 
seines Endes und der damit verbundenen Spekulationen über die Zukunft des einstigen westlichen Blocks zu verstehen. ${ }^{25}$ Insofern war sie normativ, weil sie nach der Entstehung des vermeintlich siegreichen Gesellschaftsentwurfs fragte, und sie bleibt selektiv, weil sie sich nur für westliche Einflüsse und Ordnungsmodelle interessiert. Hervorzuheben ist: Die Historiografie zur Bundesrepublik hat - beginnend mit dem Interesse für die alliierte Besatzungspolitik, die Re-education, dann die Amerikanisierung und die Westernisierung - die Nationalgeschichte schon lange vor dem Transnationalen oder Global Turn aufgebrochen. Wandlungsprozesse in der westdeutschen Gesellschaft ließen sich ohne Berücksichtigung transnationaler Faktoren nicht hinreichend erklären. Und: Der Westen der Westernisierung wurde eben nicht normativ gesetzt, sondern als historisch kontingenter Quellenbegriff verstanden, dessen Bedeutung es überhaupt erst herauszuarbeiten galt. ${ }^{26}$ Das ist der fundamentale Unterschied zu Winkler, dessen Arbeit man vielmehr als Beitrag zur Westernisierung des deutschen Geschichtsbilds im Dienste der nationalen Identität lesen kann - und weniger als Erklärung eben dieses historischen Prozesses.

Westernisierung bezog sich in den Tübinger Forschungen vor allem auf die Etablierung eines spezifischen Verständnisses von Westlichkeit in der Bundesrepublik der 1960er Jahre, wobei die empirischen Studien teils bereits im späten 19. Jahrhundert ansetzten. ${ }^{27}$ Wie in den Arbeiten zur Amerikanisierung ging es darum, Wandlungsprozesse in der westdeutschen Nachkriegsgesellschaft zu erklären. Akzentuierte Amerikanisierung in Doering-Manteuffels Sicht einen weitgehend einseitigen Transfer und untermauerte gewollt oder ungewollt den Eindruck auch kultureller US-amerikanischer Dominanz innerhalb des westlichen Blocks im Kalten Krieg, setzte er den Begriff der Westernisierung ausdrücklich hiervon ab: Er bezeichne „die allmähliche Herausbildung einer gemeinsamen Werteordnung in den Gesellschaften diesseits und jenseits des Nordatlantik“, die bereits im 19. Jahrhundert begann und stets ausdrücklich zwar nicht als globaler, aber als transnationaler Austauschprozess und interkultureller Transfer zwischen Gesellschaften des nordatlantischen Raums zu verstehen war. $^{28}$

${ }^{25}$ Vgl. zu den Debatten über die Identität des Westens in den 1990er Jahren David Gress, From Plato to NATO. The Idea of the West and Its Opponents, New York u. a. 1998, hier S. 467-502.

${ }^{26}$ Vgl. Riccardo Bavaj/Martina Steber (Hrsg.), Germany and „The West“. The History of a Modern Concept, New York/Oxford 2015.

27 Vgl. Michael Hochgeschwender, Freiheit in der Offensive? Der Kongreß für kulturelle Freiheit und die Deutschen, München 1998; Julia Angster, Konsenskapitalismus und Sozialdemokratie. Die Westernisierung von SPD und DGB, München 2003; Gudrun Kruip, Das „Welt“-„Bild“ des Axel Springer Verlags. Journalismus zwischen westlichen Werten und deutschen Denktraditionen, München 1999, und Thomas Sauer, Westorientierung im deutschen Protestantismus? Vorstellungen und Tätigkeit des Kronberger Kreises, München 1999.

${ }^{28}$ Anselm Doering-Manteuffel, Westernisierung. Politisch-ideeller und gesellschaftlicher Wandel in der Bundesrepublik bis zum Ende der 60er Jahre, in: Axel Schildt/Detlef Siegfried/ Karl Christian Lammers (Hrsg.), Dynamische Zeiten. Die 60er Jahre in den beiden deutschen Gesellschaften, Hamburg 2000, S.311-341, hier S.314. Aus amerikanischer Sicht in dieser Perspektive vgl. Mary Nolan, The Transatlantic Century. Europe and America, 18902010, Cambridge 2012. 
Damit unterschied sich das Konzept auch von Herberts Erklärungsmodell der Liberalisierung. Herbert verzahnte die mit Liberalisierung umschriebenen Veränderungen in der politischen Kultur, den Wandel von Mentalitäten, Wertedispositionen, Einstellungen und Lebensweisen der westdeutschen Gesellschaft mit der Modernisierungstheorie. ${ }^{29}$ Die kulturelle Modernisierung der 1960er und auch der 1970er Jahre war dann als endlich gelungene Anpassung an jene wirtschaftliche und technische, politische und soziale Modernisierung zu begreifen, die sich in Deutschland erst in der Bundesrepublik verdichtete und den Höhebeziehungsweise Endpunkt der industriellen Hochmoderne markierte. Das war nicht von der Ideen-, sondern von der Sozialgeschichte her gedacht, und ließ sich prinzipiell als endogener gesellschaftlicher Prozess beschreiben.

Der Westernisierungs-Ansatz betont hingegen den transnationalen Austausch von Ideen und Ordnungsvorstellungen, denen darüber hinaus gesellschaftliche und politische Gestaltungsmacht zugeschrieben wird. Insofern ist es folgerichtig, dass die Westernisierung im Zeitbögen-Entwurf eine zentrale Stellung einnimmt. Aber inwiefern führt dies zu einer normativen und teleologischen Verengung? Das Problem, das Hoeres umtreibt, scheint weniger im Westernisierungskonzept angelegt zu sein, sondern darin zu liegen, wie Doering-Manteuffel es in den Zeitbögen füllt. Das mag teils der Kürze seines Aufsatzes geschuldet sein. Trotzdem sehe ich drei Aspekte, die in ihrer Kombination eine Verengung der Westernisierung suggerieren: Erstens eine Gleichsetzung von „westlich“ und „liberal“, wobei, zweitens, der Begriff „westlich“ analytisch und normativ definiert wird - gebündelt im Konstrukt des „angloatlantischen Ordnungsmodells“ -, und drittens die Zuspitzung der Gesamtinterpretation der deutschen Zeitgeschichte auf das Ringen zwischen Liberalismus und Antiliberalismus.

\section{Westlich = liberal?}

In den Zeitbögen fallen „westlich“ und „liberal“ in eins: Dreimal im 20. Jahrhundert habe „das Ordnungsmodell des liberalen Westens“ über einen Gegner triumphiert, der den Anspruch erhoben hatte, es niederzuringen: 1918, 1945 und $1989 / 90 .{ }^{30}$ Kann man aber, sowohl für die Zwischenkriegszeit als auch für die Zeit des Kalten Kriegs, von einem kohärenten („angloatlantischen“) Ordnungsmodell des liberalen Westens sprechen? Genügt es, dieses im Sinne der Totalitarismustheorie aus einem Antagonismus zwischen Liberalismus und Antiliberalismus herzuleiten? Sind „westliche“ Normen als deckungsgleich mit „liberalen“ zu begreifen? Und welchen Platz haben in diesem Arrangement der nationalsozialistische Antikommunismus und der Antifaschismus der DDR? Das „westlich-liberale“, „angloatlantische Ordnungsmodell“ charakterisiert Doering-Manteuffel folgendermaßen: Universalismus, Tradition der Aufklärung, staats- und völkerrechtliche

\footnotetext{
${ }^{29}$ Vgl. Ulrich Herbert, Liberalisierung als Lernprozeß. Die Bundesrepublik in der deutschen Geschichte - eine Skizze, in: Ders. (Hrsg.), Wandlungsprozesse in Westdeutschland. Belastung, Integration, Liberalisierung 1945-1980, Göttingen 2002, S. 7-49.

${ }^{30}$ Doering-Manteuffel, Deutsche Geschichte, S. 324.
} 
Ordnung auf Basis „liberaler“ Normen, „liberaler“ Fortschrittsbegriff, Denken in historischer Entwicklung, parlamentarische Demokratie, Rechtsstaat, Freiheit des Individuums und Marktwirtschaft. ${ }^{31}$ In einem neueren Aufsatz spricht er vom dreimaligen Triumph des angloatlantischen Liberalismus, den er so definiert: „wirtschaftliche Freiheit des Einzelnen in der Marktgesellschaft, staatsbürgerliche Selbstbestimmung und die politische Freiheit in der parlamentarischen Demokratie sowie die Gleichheit aller Staatsbürger im Rahmen des Rechtsstaats“. ${ }^{32}$

Zum einen privilegiert diese Definition die Rechts- und Wirtschaftsordnung gegenüber anderen Dimensionen der Gesellschaftsordnung; über soziale Strukturen, Lebensstile und Einstellungen sowie die Rolle von Wissenschaft, Technik und Konsum in der modernen Gesellschaft des 20. Jahrhunderts ist damit wenig ausgesagt. Zum anderen fehlt eine für das 20. Jahrhundert zentrale Dimension von Staatlichkeit, nämlich der Sozial- und Interventionsstaat. Etwas aus dem Blick gerät damit, wie wichtig der Beitrag nicht genuin liberaler Bewegungen und Denktraditionen sowie die Liberalismuskritik, die uns in den Zeitbögen nur in Gestalt des Antiliberalismus entgegentritt, seit dem letzten Drittel des 19. Jahrhunderts für die Ausformung „westlicher“ und demokratischer Selbstverständnisse waren: Sozialismus und Sozialdemokratie, Konservatismus, katholische Soziallehre, christliche Demokratie. ${ }^{33}$ Zwar sieht Doering-Manteuffel nicht über rivalisierende ideelle Strömungen wie etwa die egalitär-etatistische und die individualistisch-freiheitliche Tradition hinweg, die aus dem Aufklärungsliberalismus des späten 18. Jahrhunderts bis ins 20. Jahrhundert hineinreichten. ${ }^{34}$ Doch nicht nur im innerliberalen, sondern vor allem im ideellen Konflikt liberaler mit nichtliberalen und liberalismuskritischen Strömungen - und nicht allein mit antiliberalen (totalitären) - entwickelte sich ein wesentlich vielfältigeres Verständnis von Westlichkeit und einer „westlich“-demokratischen Staats- und Gesellschaftsordnung, als es in den Zeitbögen definiert wird. ${ }^{35}$ Wird „westlich“ als Synonym zu „liberal“ verstanden, reduziert das den Begriff von Westlichkeit auf eine rechtsstaat-

31 Ebenda, S. 330-332.

32 Anselm Doering-Manteuffel/Jörn Leonhard, Liberalismus im 20. Jahrhundert. Aufriss einer historischen Phänomenologie, in: Dies. (Hrsg.), Liberalismus im 20. Jahrhundert, Stuttgart 2015, S. 13-32, hier S. 18.

${ }^{33}$ Vgl. Michael Hochgeschwender, Was ist der Westen? Zur Ideengeschichte eines politischen Konstrukts, in: Historisch-Politische Mitteilungen 11 (2004), S. 1-30, hier S. 15-19.

${ }^{34}$ Vgl. Doering-Manteuffel, Westernisierung, in: Schildt/Siegfried/Lammers (Hrsg.), Dynamische Zeiten, S.314, und ders., Wie westlich sind die Deutschen? Amerikanisierung und Westernisierung im 20. Jahrhundert, Göttingen 1999, S. 13 f.

35 Vgl. Gress, From Plato to NATO, S. 407-466, der zeigt, dass das Ringen um das Selbstverständnis des Westens stets von Konflikten und konkurrierenden Konzeptionen geprägt war. Allein zwischen dem Ende des Zweiten Weltkriegs und den späten 1960er Jahren etablierte sich kurzzeitig eine hegemoniale, antikommunistische Identitätserzählung des Westens als kulturelle Wertegemeinschaft, mit der die politische und militärische Gemeinschaft der „Atlantic Community“ unterfüttert wurde. Zur Hochphase dieses im „Atlanticism“ gebündelten Selbstverständnisses in der US-Außenpolitik vgl. Kenneth Weisbrode, The Atlantic Century. Four Generations of Extraordinary Diplomats Who Forged America's Vital Alliance with Europe, Cambridge 2009, hier S. 83-202. 
liche, demokratische und marktwirtschaftliche Ordnung, deren Kern allein mit den Leitbegriffen Marktwirtschaft und Demokratie und dem abstrakten Vokabular der politischen und Rechtstheorie beschrieben werden kann. Nach einer solchen Definition ist dann allerdings auch die neoliberale Ordnung des späten 20. und frühen 21. Jahrhunderts, die Doering-Manteuffel im dritten Zeitbogen als Bruch mit dem „angloatlantischen Modell“ charakterisiert, als liberale, westliche Ordnung klassifizierbar.

Über den Umgang dieser Ordnung mit Fragen gesellschaftlicher Chancengleichheit, sozialer Ungleichheit oder sozialer Gerechtigkeit, über die damit verbundenen ökonomischen und politischen Kräfteverhältnisse sowie die Rolle des Sozial- und Interventionsstaats erfahren wir dabei wenig. Dem „angloatlantischen Modell“ scheinen damit eine Reihe ideeller und institutioneller Ordnungskomponenten zu fehlen, die für die demokratischen Staaten im nordatlantischen Raum seit den 1930er Jahren konstitutiv waren. Doering-Manteuffel versucht dieses Problem zu lösen, indem er die liberale Ordnung des atlantischen Modells als Sozialliberalismus charakterisiert, der bereits direkt nach dem Ersten Weltkrieg das deutsche wie das internationale Erscheinungsbild des Liberalismus geprägt habe. ${ }^{36}$ So wirkt sein ,angloatlantisches Modell“ recht statisch und homogen; nationale Unterschiede - insbesondere zwischen dem deutschen und US-amerikanischen Liberalismus - und Transformationen innerhalb des Liberalismus werden angedeutet, aber der binären Opposition Liberalismus versus Antiliberalismus untergeordnet. Durch die Konzentration auf diese Dichotomie geraten andere, nicht-totalitäre Strömungen ebenso in den Hintergrund wie die einer modernen Gesellschaft inhärente Dynamik, die sich nicht zuletzt in den permanenten Aushandlungsprozessen und Konflikten über den Gehalt jener äußerst umkämpften und umstrittenen Kernbegriffe wie Freiheit, Fortschritt, Selbstbestimmung oder Gleichheit äußerte. Mit der tendenziell statischen Definition wird das alte analytische Konzept der Westernisierung, das die Entstehung eines bestimmten Verständnisses von Westlichkeit und das, was zu bestimmten Zeiten als westlich galt, erst erklären sollte, normativ umdefiniert und kann als teleologischer Prozess missverstanden werden. Der Westen der Westernisierung - dort noch Quellenbegriff und historisch kontingentes Konstrukt - mutiert zu dem einen, „liberalen“ Westen, in dem man ankommen kann. Dem Autor scheint dieses Problem bewusst zu sein, denn er arbeitet mal mit, mal ohne Anführungszeichen: Der Begriff des Westens changiert in den Zeitbögen zwischen Quellenbegriff („Westen“ in Anführungsstrichen), geographischer Bezugsgröße (moderne Industriegesellschaften im „angloatlantischen“ Raum), politischer Handlungseinheit (Institutionen- und Beziehungsgefüge der Staaten des westlichen Blocks im Kalten Krieg) und normativ-analytischem Idealtypus („angloatlantisches Modell“), der „westlich“ mit „liberal“ gleichsetzt und darunter parlamentarische Demokratie, staatsbürgerliche Gleichheit, individuelle Freiheitsrechte und freie Marktordnung versteht.

Neben dem für die deutsche Zeitgeschichte - und auch für den unten noch zu diskutierenden Konsensliberalismus - so wichtigen Konzept der sozialen Demo-

${ }^{36}$ Vgl. Doering-Manteuffel, Deutsche Geschichte, S. 330. 
kratie, ihrer liberalismuskritischen Trägergruppen und dem Sozial- und Wohlfahrts- beziehungsweise Interventionsstaat rückt damit eine weitere Facette gesellschaftlicher Transformation im Westdeutschland der 1960er Jahre und frühen 1970er Jahre in den Hintergrund - und zugleich, wenn man sich erlaubt, analytisch und normativ zu sein, eine in „westlich-liberalen“ Gesellschaften kaum zu unterschätzende Kraft sozialen Wandels: nämlich Konflikt und Dissens. Bernd Greiner unterstreicht diese Facette mit Blick auf die Amerikanisierung in der Bundesrepublik der 1960er Jahre: Erst jetzt ließen sich eine Emanzipation des deutschen Untertanen zum Bürger und die Aufwertung des politischen Bürgers gegenüber dem Staat beobachten; Politik und Öffentlichkeit rangen um die gesellschaftliche Anerkennung des Rechts auf zivilen Widerstand, und die westdeutsche Gesellschaft lernte, Konflikt und Streit als Essenz der Demokratie, Widerspruch und Dissens als Bereicherung zu verstehen und die Grenzen der Toleranz dort zu markieren, wo dieselben bedroht erschienen. ${ }^{37}$ Diese Akzentsetzung benennt Greiner ausdrücklich als eine historisch normative: Der gesellschaftliche Umgang mit Dissens ist für ihn ein essenzielles Merkmal einer westlichen demokratischen Gesellschaft.

\section{Konsensliberalismus und „liberaler Konsens"}

Doering-Manteuffels Akzentuierung ist eine andere, und sein normativer Standpunkt bleibt unausgesprochen. Hoeres hatte hier vermutlich die richtige Intuition, konnte seine Kritik aber nicht richtig auf den Punkt bringen. Doering-Manteuffel betont mehrfach das Ende eines „liberalen Konsenses" in der Bundesrepublik seit den 1970er Jahren, dessen Aufkündigung für ihn bereits mit den Konflikten um 1968 begann. ${ }^{38}$ Aus seinen Formulierungen könnte man einen Ausdruck des Bedauerns lesen. So ist etwa von einem „Wandel in der Politik vom Primat des sozialen Konsenses zum Primat des Subjektivismus“ die Rede, ${ }^{39}$ und es heißt:

„Ein neuer Individualismus in der Konsumentenkultur brach sich Bahn. Es war ein Anspruch auf Freiheit des Einzelnen nach Maßgabe des privaten Interesses. [...] Die Freiheit des Konsenses im Rahmen von parlamentarischer Demokratie und sozialer Marktwirtschaft trat zurück hinter den Anspruch des konsumistischen Individuums auf seine eigene Freizügigkeit im Rahmen einer freiheitlichen Ordnung, die ganz selbstverständlich als dauerhaft gültig betrachtet wurde. “40

\footnotetext{
${ }^{37}$ Vgl. Bernd Greiner, „Test the West“. Über die „Amerikanisierung“ der Bundesrepublik Deutschland, in: Mittelweg 366 (1997), S. 4-40, hier S. 34-36.

${ }^{38}$ Vgl. Doering-Manteuffel, Deutsche Geschichte, S. 344 u. Zitat S. 343.

${ }^{39}$ Ebenda, S. 345.

${ }^{40}$ Ebenda, S. 344.
} 
Subjektivismus, Individualismus und Konsumismus erscheinen als pejorativ konnotierte Termini, wie sie seit dem 19. Jahrhundert zum Repertoire konservativer Kulturkritik gehören. Hier spricht aus dem Autor offenbar ein Kulturpessimist, der dem Golden Age einer idealisierten „Konsenskultur“41 nachzutrauern scheint, die gesellschaftlich in der kurzen Periode zwischen der Mitte der 1960er und Mitte der 1970er Jahre verankert und politisch an einer Schnittstelle zwischen progressivem Konservatismus, sozialliberalem Liberalismus und zentristisch-moderater Sozialdemokratie zu verorten sein könnte.

Worin bestand dieser „liberale Konsens“? In der Deutung der Zeitbögen wurde er nach dem Zweiten Weltkrieg in Gestalt des „angloatlantischen Modells“ liberaler Westlichkeit aus den USA in die Bundesrepublik importiert. Wenn ich Doering-Manteuffel richtig verstehe, vereinten sich nun im Prinzip die deutsche beziehungsweise europäische und die amerikanische Spielart des Sozialliberalismus. Denn die Wurzeln des „liberalen Konsenses“ verortet Doering-Manteuffel in den USA, zunächst im New Deal: In diesem Zusammenhang ist vom „New Deal Konzept des liberalen und sozialen Konsenses“ die Rede, worunter er ein neuartiges Zusammenspiel von kapitalistischer Wirtschaft, politischer Demokratie und gesellschaftlicher Freiheit versteht. ${ }^{42}$ Hierfür könnte man, wie weiter unten noch zu zeigen ist, auch den Begriff der New Deal Order verwenden. Die Ausdrücke Konsensliberalismus und „liberaler Konsens“ benutzt Doering-Manteuffel synonym: „Mit dem Ende des Booms kam auch das Ende des liberalen Konsenses“, beziehungsweise „Der Konsensliberalismus aus der Epoche des Booms war [Mitte der 1970er] zum Merkmal des Ancien régime geworden“.43

Doering-Manteuffel übernimmt damit Definitionen der frühen Westernisierungsforschung, die zahlreiche Anregungen aus der US-amerikanischen Historiografie bezog und unter Konsensliberalismus folgendes verstand: erstens in geistesgeschichtlicher Perspektive eine neuartige politische Leitideologie, die sich aus Elementen des amerikanischen Reformliberalismus, Pragmatismus, Keynesianismus und Wilsonianischem Internationalismus zusammensetzte. Die Träger dieses Ideenguts gruppierten sich in den 1930er Jahren um die Vordenker des New Deal und in den 1940er und 1950er Jahren um Intellektuellenzirkel wie die New York Jewish Intellectuals, die es dann über transatlantische Netzwerke wie den Kongress für kulturelle Freiheit oder die Rockefeller Foundation nach Westeuropa und Westdeutschland trugen. Zweitens verweist Konsensliberalismus auf einen Strang der amerikanischen Historiografie, der im Zeichen des Kalten Kriegs in den 1950er Jahren ein hegemoniales Narrativ zu etablieren suchte, mit dem die amerikanische Geschichte seit der Kolonialzeit auf die konsensliberale Leitideologie hingeschrieben werden sollte. ${ }^{44}$ Nicht soziale und wirtschaftliche Konflikte, sondern Konsens prägten demnach die Geschichte der USA. Damit bezog sich Konsensliberalismus drittens auf einen (vermeintlichen) Konsens in der amerika-

\footnotetext{
${ }^{41}$ Ebenda, S. 345.

42 Ebenda, S. 338.

43 Ebenda, S. 341 u. S. 343.

${ }^{44}$ Vgl. mit diesen beiden Akzenten Hochgeschwender, Freiheit in der Offensive, S. 68-86.
} 
nischen Gesellschaft der 1950er Jahre über die Grundlagen des American way of life in einem neuen Zeitalter des Überflusses, in dem Klassengegensätze und politisch-ideologische Konflikte durch stetes wirtschaftliches Wachstum und zunehmenden gesellschaftlichen Wohlstand aufgelöst würden. Die ideellen Elemente des Konsensliberalismus spiegelten sich viertens in den wirtschaftspolitischen Ordnungsvorstellungen amerikanischer Gewerkschaften wider, die diese Vorstellungen über die transatlantischen Netzwerke der Arbeiterbewegung in die in den 1950er Jahren noch sozialistisch orientierte westdeutsche Sozialdemokratie transponierten. ${ }^{45}$

Besonders Julia Angster unterstrich schließlich fünftens die Verflechtung von Konsensliberalismus mit dem, was in der amerikanischen Historiografie als New Deal Order charakterisiert wird. ${ }^{46}$ Dieser Zusammenhang wird in den Zeitbögen eher en passant erwähnt, scheint aber geeignet, die konzeptionelle Programmatik einer „Gesellschaftsgeschichte handlungssteuernder Ideen [...] und Ordnungskonzepte ${ }^{\text {"47 }}$ zu akzentuieren. Das Konzept der New Deal Order ist etwas anders gelagert als das Konstrukt des Konsensliberalismus und bezieht sich auf eine Neudefinition der Kräfteverhältnisse in der politischen Ökonomie der USA zwischen den 1930er und frühen 1970er Jahren, auf eine institutionelle Neuordnung der Beziehungen zwischen Staat, Wirtschaft und Gesellschaft - die für die USA überhaupt erst eine Aktualisierung des Bundesstaats in der Sozial- und Wirtschaftspolitik bedeutete - und schließlich auf eine spezifische Struktur der Wählerschaft und Allianzen von Interessengruppen im Umfeld der Demokratischen Partei. ${ }^{48}$ Berücksichtigt man also die politischen Machtverhältnisse und die Politiknetzwerke mit ihren Interessengruppen und Ideengebern, lassen sich die Ideengeschichte und die Geschichte der politischen Ökonomie, deren Bedeutung Doering-Manteuffel auch für Bogen drei zum Zusammenhang zwischen Globalisierung und Neoliberalismus andeutet, miteinander in Beziehung setzen. Jedoch: Kann man den Konsensliberalismus als „Spielart des Liberalismus" ${ }^{* 49}$ klassifizieren und mit einem gesellschaftlichen Konsens oder gar einer „Konsenskultur“ in der Bundesrepublik identifizieren? Mein Eindruck ist, dass die westdeutsche Nachkriegsgeschichte hier im Sinne des auf die amerikanische Nachkriegszeit gemünzten consensus-Narrativs zu glatt gebügelt wird.

Der vermeintliche liberal consensus beziehungsweise postwar consensus in den USA zwischen 1945 und 1965, der sich in Doering-Manteuffels Diagnose einer „Konsenskultur“ und eines „sozialen Konsenses“ in der Bundesrepublik vor 1968 und dem Ende des Booms wiederfindet, war ein lange gebräuchliches Narrativ

\footnotetext{
${ }^{45}$ Vgl. zu diesen beiden Aspekten Angster, Konsenskapitalismus und Sozialdemokratie, S. 3971.

${ }^{46}$ Vgl. ebenda, S. 71-98.

${ }^{47}$ Vgl. Doering-Manteuffel, Deutsche Geschichte, S. 324.

48 Vgl. Jefferson Cowie, The Great Exception. The New Deal \& the Limits of American Politics, Princeton 2016, und Steve Fraser/Gary Gerstle (Hrsg.), The Rise and Fall of the New Deal Order, 1930-1980, Princeton 1989.

49 Doering-Manteuffel, Westernisierung, in: Schildt/Siegfried/Lammers (Hrsg.), Dynamische Zeiten, S. 321.
} 
der amerikanischen Historiografie, die von den ersten beiden Nachkriegsjahrzehnten das harmonische Bild eines breiten, antikommunistischen gesellschaftlichen Wertekonsenses, des sozialen Friedens und zunehmenden Wohlstands in allen Schichten zeichnete, als Fragen sozialer Ungleichheit an Bedeutung verloren und sich ideologische und Klassengegensätze auflösten. Dieses Narrativ schrieb einen zeitgenössischen Diskurs der Nachkriegszeit fort, der die sozialen Konflikte in einer ethnisch und religiös heterogenen Gesellschaft hinter einer einenden Erzählung nationaler Identität verschwinden ließ. Den Kitt dieser in den 1940er und 1950er Jahren forcierten culture of consensus bildete die Beschwörung von Idealen der amerikanischen Demokratie im Angesicht des europäischen Faschismus und der kommunistischen Bedrohung, deren antireligiösem Kollektivismus die USA die Freiheit des Einzelnen, das ökonomische Ideal des free enterprise, den Glauben an Gott und den amerikanischen Universalismus gegenüberstellten. ${ }^{50}$ Damit verbanden sich in den 1950er Jahren ein Klima der Selbstgefälligkeit und Paranoia und eine eigentümliche Selbstgleichschaltung des intellektuellen und politischen Diskurses. Die „Konsenskultur“, hochgehalten von Politikern, Wirtschaftseliten und Massenmedien, hatte einen repressiven Charakter; Dissens und Kritik wurden als Abweichen stigmatisiert, und politische Opposition reduzierte sich im nationalen Schulterschluss gegen den Kommunismus auf ein Minimum. ${ }^{51}$ Wie die abrupten, von der weißen Mittelschicht und dem politischen Establishment irritiert wahrgenommenen Rassenunruhen seit den frühen 1960er Jahren, die Radikalisierung der Bürgerrechts- und Studentenbewegung und die Vielfalt neuer sozialer und politischer Bewegungen zeigten, war der vermeintliche gesellschaftliche Konsens ausgesprochen fragil und einseitig. Bis in die 1990er Jahre wirkte das consensus-Narrativ indes in der Historiografie nach, die gemäß dieser Standarderzählung den Druck von links und den darauf folgenden konservativen backlash für einen Zerfall des Konsenses verantwortlich machte. Gesellschaftliches Konfliktpotenzial, politische Bruchlinien und die fortgesetzte Diskriminierung der afroamerikanischen Bevölkerung in den Nachkriegsjahrzehnten wurden durch das consensus-Narrativ ebenso überdeckt wie die Neuformierung des amerikanischen Konservatismus nach 1945 und eines im Umfeld der Republikanischen Partei verbreiteten, vollkommen anderen Verständnisses von Liberalismus als jenem der New Deal- und Great Society-Liberalen. ${ }^{52}$

Diese letzte Unterscheidung ist ungemein wichtig und führt zu einem terminologischen Problem, das in den Zeitbögen nicht thematisiert wird: In den 1930er

\footnotetext{
${ }^{50}$ Vgl. Wendy L. Wall, Inventing the „American Way“. The Politics of Consensus from the New Deal to the Civil Rights Movement, Oxford 2008.

${ }^{51}$ Vgl. Godfrey Hodgson, America in Our Time. From World War II to Nixon. What Happened and Why, New York 1976, hier S. 67-98.

${ }^{52}$ Vgl. Gary Gerstle, Race and the Myth of the Liberal Consensus, in: The Journal of American History 82 (1995), S.579-586. Zum Konservatismus als Stiefkind des „consensus“-Narrativs vgl. Alan Brinkley, Liberalism and Its Discontents, Cambridge 1998, S.277-297, und Kim Phillips-Fein, „If Business and the Country Will Be Run Right“. The Business Challenge to the Liberal Consensus, 1945-1964, in: International Labor and Working-Class History 72 (2007), S. 192-215.
} 
Jahren wurde der Ausdruck liberal im Amerikanischen auf eine heute noch gültige Weise umdefiniert, die in einem Gegensatz zum europäischen Verständnis von liberal im Sinne des klassischen Liberalismus steht und auch nicht identisch ist mit dem, was in der deutschen Diskussion ${ }^{53}$ als Sozialliberalismus im Sinne eines reformierten, postklassischen Liberalismus verstanden wird. In ideen- und politikgeschichtlicher Perspektive bezeichnet liberal im Amerikanischen seit den 1930er Jahren eine Schnittmenge aus reformliberalen, progressiven, sozialdemokratischen, keynesianischen, technokratischen und vor allem etatistischen Orientierungen. Die Ausdehnung des amerikanischen Interventionsstaats seit den 1960er Jahren, die mit der reformpolitischen Agenda der Great Society verknüpft war, jedoch auch den Bereich wirtschaftspolitischer Regulierung betraf, wird in der Historiografie als Hochphase des amerikanischen liberalism charakterisiert und die Planungseuphorie der 1960er Jahre als liberal hour, als Sternstunde des Liberalismus. ${ }^{54}$

Diese genuin amerikanische Neudefinition, die ausdrücklich nicht genuin liberale sowie liberalismuskritische Elemente umfasste, geht auf die 1930er Jahre und die Auseinandersetzungen zwischen den New Deal-Demokraten um Franklin D. Roosevelt und den geschlagenen Republikanern um Herbert Hoover zurück. Den Demokraten gelang es, den Republikanern den Terminus liberalzu entreißen und im Sinne eines säkularen und etatistischen Progressivismus neu zu besetzen: Liberal bedeutete unter dem Eindruck der Wirtschaftskrise eine aktive Rolle des Staats zu unterstützen, um durch die Verbürgung sozialer Sicherheit und sozialer Rechte die ökonomische und staatsbürgerliche Freiheit aller gesellschaftlichen Gruppen in der Massendemokratie zu garantieren, wobei die Afroamerikaner erst mit den Bürgerrechtsgesetzen von 1964 und 1965 explizit eingeschlossen waren. Dies richtete sich ausdrücklich gegen den Laissez-Faire-Liberalismus der Republikanischen New Deal-Opposition, die unter „liberal“ die Betonung individueller Freiheitsrechte und die Beschränkung des Staats auf das Nötigste verstanden wissen wollte..$^{55}$ Den welfare liberals sei es so gelungen, sich als legitime Erben in die Kontinuität der liberalen amerikanischen Tradition zu stellen. ${ }^{56}$ Nach dem Ver-

${ }^{53}$ Vgl. etwa Jens Hacke, Selbstkritik und Selbstzweifel. Zur Krise des liberalen Denkens in der Zwischenkriegszeit, in: Ewald Grothe/Ulrich Sieg (Hrsg.), Liberalismus als Feindbild, Göttingen 2014, S. 153-182.

${ }^{54}$ Vgl. Sidney M. Milkis/Jerome M. Mileur (Hrsg.), The Great Society and the High Tide of Liberalism, Amherst 2005, und G. Calvin Mackenzie/Robert Weisbrot, The Liberal Hour. Washington and the Politics of Change in the 1960s, New York 2008.

${ }^{55}$ Vgl. Maurizio Vaudagna, Conservative Critics of the New Deal in the 1930s. Towards Authoritarian Europeanization?, in: Ders. (Hrsg.), The Place of Europe in American History. Twentieth-Century Perspectives, Turin 2007, S. 267-321, hier S.299f. Auf diese Zeit geht dann schließlich auch die amerikanische Dichotomie zwischen „liberal“ und „conservative“ zurück.

${ }^{56}$ Vgl. Michael P. Zuckert, On Constitutional Welfare Liberalism. An Old-Liberal Perspective, in: Ellen Frankel Paul/Fred D. Miller Jr./Jeffrey Paul (Hrsg.), Liberalism. Old and New, Cambridge 2007, S. 266-288, hier S. 275. Anders als in Deutschland bildet die Auseinandersetzung über die richtige Auslegung der im klassischen Liberalismus verwurzelten Verfassungsordnung und darüber, was die liberale Tradition der Vereinigten Staaten ausmacht, seit 
ständnis dieses New Deal- und Great Society-liberalism kam dem Staat die Rolle des Agenten gesellschaftlichen Fortschritts und ökonomischen Wohlstands zu.

\section{VI. „Konsenskultur": Das Golden Age der Bundesrepublik?}

Wie es scheint, muss Doering-Manteuffel die Terminologie bewusst offen lassen, denn nur mit Hilfe des unscharfen Konsensliberalismus und der Gleichsetzung von (amerikanisch) liberal und (deutsch) liberal können sämtliche demokratisch orientierten (antitotalitären) Strömungen und Ordnungsvorstellungen - also auch sozialdemokratische und konservative - dem Liberalismus zugeschlagen und in den Widerstreit zwischen Liberalismus und Antiliberalismus als master narrative der deutschen Geschichte des 20. Jahrhunderts eingepasst werden. Konsensliberalismus, „liberaler Konsens“ und das consensus-Narrativ der amerikanischen Historiografie verschmelzen zu einer vermeintlichen „Konsenskultur“, die an Hobsbawms Stilisierung des Golden Age der keynesianischen Nachkriegsjahrzehnte erinnert. ${ }^{57}$ Das Golden Age der Bundesrepublik scheinen jene Jahre gewesen zu sein, in denen sich alle drei politischen Parteien auf dem festen Boden des Grundgesetzes einträchtig zum „angloatlantischen Modell“ der liberalen, westlichen Demokratie bekannten, nach der Zeitbögen-Definition also zur freiheitlich-demokratischen Grundordnung und zur Marktwirtschaft. Das könnte mit der „Konsenskultur“ gemeint sein, auf die sich der zweite Zeitbogen narrativ zuspitzt - eine deutsche Fassung des amerikanischen consensus-Narrativs gewissermaBen.

Dieses eigentlich historisch spezifische „angloatlantische Modell“ verkörpert für Doering-Manteuffel somit gleichzeitig den normativen Idealtyp liberaler Westlichkeit, und der Konsens über dieses Verständnis bildet den historisch-normativen Maßstab, vor dem die Entwicklungen des dritten Zeitbogens tendenziell nur als Auflösungs- oder Verlusterzählung dargestellt werden können. Das ist eine ähnliche Interpretation wie die einflussreiche Deutung von Hobsbawm, dessen nun schon mehr als zwanzig Jahre alte Darstellung immer noch einige interessante Anregungen enthält. Dies betrifft besonders die Dimension der internationalen politischen Ökonomie, ohne die sich die Zeitgeschichte seit den 1970er Jahren in Zukunft kaum schreiben lassen wird, und eine Leerstelle der zeitgeschichtlichen Forschung, die bei Doering-Manteuffel in der Formulierung „Marktwirtschaft und Demokratie“" versteckt ist: ${ }^{58}$ der Nexus zwischen Kapitalismus und Demokratie und dessen Transformation seit $1945 .{ }^{59}$ Hobsbawm be-

\footnotetext{
dem 18. Jahrhundert eine zentrale Achse politischer, intellektueller und gesellschaftlicher Debatten. Die Berufung auf die im Freiheitsbegriff gebündelte liberale Tradition ist im gesamten politischen Spektrum der USA von der extremen Linken bis zur radikalen Rechten anzutreffen und zählt zum Kernbestand nationaler Identität.

57 Vgl. Hobsbawm, Zeitalter der Extreme, hier S. 324-362.

${ }^{58}$ Doering-Manteuffel, Deutsche Geschichte, S. 348.

${ }^{59}$ Vgl. Wolfgang Streeck, Re-Forming Capitalism. Institutional Change in the German Political Economy, Oxford 2009, und ders., Gekaufte Zeit. Die vertagte Krise des demokratischen Kapitalismus, Berlin 2013.
} 
schrieb die Phase zwischen den späten 1940er und den frühen 1970er Jahren als goldenes Zeitalter der Wohlstandsexpansion, ermöglicht durch technologische Entwicklungen sowie eine Internationalisierung und Globalisierung der Wirtschaft. Der Wohlfahrtsstaat förderte den Konsum, vor allem aber - das ist entscheidend - wurde der Kapitalismus in den westlichen Staaten bewusst in seiner Substanz restrukturiert und reformiert: Indem der demokratische Staat in den 1940er Jahren die steuernde Rolle in der wirtschaftlichen Modernisierung übernahm, gelang es, wirtschaftlichen Liberalismus und soziale Demokratie miteinander zu vereinen ${ }^{60}$ Hobsbawm betonte die zentrale Rolle der USA für die Stabilität der internationalen Wirtschaftsordnung, in die die nationalen Ökonomien und Regierungen eingebunden waren. Bereits seit den 1960er Jahren lösten sich die Aktivitäten der transnationalen Wirtschaft vom Nationalstaat ab, und es begann eine Ära langfristiger Schwierigkeiten, neuer Probleme und anhaltender Krisen in der kapitalistischen Welt. Massenarbeitslosigkeit, Konjunkturkrisen, steigende Staatsausgaben sowie eine neue Entfesselung des Laissez-Faire-Liberalismus besiegelten Hobsbawm zufolge in den 1970er Jahren das Ende des goldenen Zeitalters.

\section{VII. „Nach dem Boom”: Krise und Auflösung?}

Auch Doering-Manteuffel charakterisiert seinen dritten Zeitbogen, die Jahre seit den frühen 1970er Jahren, im Modus einer Krisendiagnose. Zu dieser Periode hat er sich, meist gemeinsam mit Lutz Raphael, seit 2008 in verschiedenen Beiträgen geäußert und die These des „Strukturbruchs“ und des „sozialen Wandels revolutionärer Qualität“ präzisiert. ${ }^{61}$ Schon 2011 unterstrichen Doering-Manteuffel und Raphael, dass zugleich nach Wohlstandsgewinnen und positiven Erfahrungen zu fragen ist, die sich für verschiedene Segmente der Gesellschaft mit dem Wandel von Arbeitswelt und Lebensbedingungen seit den 1970er Jahren verbanden. ${ }^{62}$ Für beide ist es weiterhin haltbar, von einem Epochenzusammenhang „nach dem Boom“ zu sprechen, wobei sich jedoch die zweite Hälfte der 1990er Jahre als Binnenzäsur aufdränge, da erst jetzt die für die unmittelbare Gegenwart so prägende Globalisierung sowie der weltumspannende Finanzmarktkapitalismus ihre gesellschaftliche Wirkung entfalteten. ${ }^{63}$ Neben Krisen gilt es also, Aufbrüche zu betrachten, was im Zeitbogen-Aufsatz etwas zu kurz kommt. Dort geht es um die

${ }^{60}$ Vgl. Hobsbawm, Zeitalter der Extreme, hier S. 338-350.

${ }^{61}$ Anselm Doering-Manteuffel/Lutz Raphael, Nach dem Boom. Perspektiven auf die Zeitgeschichte seit 1970, Göttingen 2008, S.10f. Weiterführend vgl. dies./Thomas Schlemmer (Hrsg.), Vorgeschichte der Gegenwart. Dimensionen des Strukturbruchs nach dem Boom, Göttingen 2016.

${ }^{62}$ Vgl. Anselm Doering-Manteuffel/Lutz Raphael, Der Epochenbruch in den 1970er-Jahren. Thesen zur Phänomenologie und den Wirkungen des Strukturwandels „nach dem Boom“, in: Knud Andresen/Ursula Bitzegeio/Jürgen Mittag (Hrsg.), „Nach dem Strukturbruch“? Kontinuität und Wandel von Arbeitsbeziehungen und Arbeitswelt(en) seit den 1970er-Jahren, Bonn 2011, S. 25-40.

${ }^{63}$ Vgl. Anselm Doering-Manteuffel/Lutz Raphael, Nach dem Boom. Neue Einsichten und Erklärungsversuche, in: Dies./Schlemmer (Hrsg.), Vorgeschichte der Gegenwart, S. 9-34. 
Auflösung des liberalen Konsenses und die Etablierung eines neuen, individualistischen und marktzentrierten Freiheitsbegriffs, der sich im Zuge sozioökonomischen und kulturellen Wandels entwickelt habe. Der industriewirtschaftliche Umbruch und die sozialkulturelle Pluralisierung hätten bewirkt, dass die Homogenität der Wiederaufbaugesellschaft mit ihrer „Chancengleichheit im Wohlstand und ihrer Konsenskultur" bis zum Ende der 1970er Jahre an Bedeutung verlor. ${ }^{64}$

Der „soziale und liberale Konsens“ geriet also gewissermaßen in den Zangengriff der pluralistischen und konsumorientierten Spaßgesellschaft, des Strukturwandels, der marktliberalen Ideologen Friedrich August von Hayek und Milton Friedman sowie ihrer politischen Erfüllungsgehilfen, die Deregulierung und Privatisierung anstießen. Der Liberalismus, der 1989/90 seinen dritten Triumph nach 1918 und 1945 verbuchen konnte, ließ bereits das neue Gewand des Neoliberalismus erkennen, der dem Markt den Vorrang vor dem Staat einräumte und das individuelle Interesse über die Belange der Gesellschaft und des Gemeinwesens stellte. ${ }^{65}$ „War das das Ende des Liberalismus in seiner für das 20. Jahrhundert charakteristischen atlantischen Spielart, das Ende des ,Westens ‘ als Verkörperung des liberalen Zusammenspiels von Marktwirtschaft und Demokratie?“, fragt Doering-Manteuffel am Ende. ${ }^{66}$

Darauf gibt es gegensätzliche Antworten. Fragt man nach dem spezifischen $\mathrm{Zu}$ sammenspiel von Marktwirtschaft und Demokratie, wird man angesichts der Entwicklungen im 21. Jahrhundert tentativ mit Ja antworten, könnte hierzu aber auf wenig historische Literatur zurückgreifen. Definiert man jedoch westlich synonym mit liberal als „angloatlantisches Modell“, dessen Kern staatsbürgerliche Gleichheit, Rechtsstaat, parlamentarische Demokratie und Marktwirtschaft bilden, muss man diese Frage wohl mit Nein beantworten. Denn wie weiter oben bereits erwähnt, wäre eine so allgemein definierte liberale Ordnung durchaus mit neoliberalen Selbstverständnissen vereinbar. Für den dritten Bogen erzeugt die terminologische Unschärfe im Begriff des Liberalen deshalb ein Abgrenzungsproblem und ein ideengeschichtliches Dilemma: Es fällt dem Autor schwer, den Neoliberalismus im Bezug zum liberalen Denken zu verorten, scheint er doch im Gegensatz zum „liberalen Konsens“ zu stehen, der sich für die Bundesrepublik möglicherweise treffender als christ- und sozialdemokratischer Konsens über eine pluralistische, soziale Demokratie und staatlich eingebettete Marktwirtschaft charakterisieren ließe. Den kündigten indes weder die Linken noch die Konservativen, sondern die Liberalen um Otto Graf Lambsdorff auf. Was sich hingegen seit den späten 1960er Jahren langsam aufzulösen begann, war die transatlantische Übereinkunft der ersten beiden Nachkriegsjahrzehnte, die nationalen Ökonomien der atlantischen Gemeinschaft in ein Gefüge transnationaler Organisationen und Regelwerke einzubetten, das in kooperativer Regulierung der Handelsbeziehungen, der Wettbewerbsbedingungen und des Währungssystems jene

\footnotetext{
${ }^{64}$ Doering-Manteuffel, Deutsche Geschichte, S. 345.

65 Vgl. ebenda.

${ }^{66}$ Ebenda, S. 348.
} 
Re-Internationalisierung der Wirtschaft erlaubte, die um 1930 unter allseitigem Protektionismus zusammengebrochen war.

Dies führt zurück zu Hobsbawm: Das goldene Zeitalter wurde ermöglicht, indem der demokratische Staat den Kapitalismus einhegte. Hobsbawm zufolge waren wirtschaftlicher Liberalismus und soziale Demokratie in der westlichen Welt bis in die 1970er Jahre vereint. Ähnlich beschrieb der Politikwissenschaftler Wolfgang Streeck Ende der 1990er Jahre die Grundkonstellation in den westlichen Demokratien im 20. Jahrhundert als Ringen zwischen liberaler und sozialer Demokratie. Im Zuge der europäischen Integration und der Internationalisierung der Wirtschaft habe sich die Balance nicht nur zugunsten der liberalen Demokratie verschoben, als transnationale Regulierungsinitiativen hinter der Marktintegration zurückstanden. Außerdem sei mit der Internationalisierung eine Stärkung der Exekutiven auf Kosten der Parlamente einhergegangen: Je mehr Entscheidungen in internationale Organisationen und transnationale Gremien verlagert wurden, desto weniger waren diese Entscheidungen der gesellschaftlichen Deliberation und parlamentarischen Mehrheitsfindung unterworfen. ${ }^{67}$ Hier schwingt ohne Zweifel eine neue Kritik an der „formalen“ statt „substanziellen“ Demokratie mit. ${ }^{68}$ Die wichtige Frage, die Hobsbawm und Streeck aufwerfen, ist die nach der Rolle des demokratischen Staats gegenüber der Wirtschaft und ihren Interessengruppen.

\section{Der demokratische Staat in der Globalisierung}

Wie sich die Rolle des (National-)Staats und die nationalen Demokratien - als gesellschaftliche wie politische Systeme - unter den Bedingungen der europäischen Integration, einer transnationalen und globalisierten Wirtschaft, der Finanzialisierung ${ }^{69}$ sowie dem Druck marktliberalen Ideenguts und wirtschaftlicher Interessengruppen seit den 1970er Jahren entwickelten, gehört wohl zu den interessantesten Fragen der zeitgeschichtlichen Forschung in den kommenden Jahren. Mit Bezug auf den Nationalstaat schlägt Hoeres in seiner Kritik an den Zeitbögen vor, Prozesse der Entnationalisierung im Zuge der deutschen Zweistaatlichkeit und der Globalisierung sowie der Renationalisierung im Kontext der Wiedervereinigung zu untersuchen. ${ }^{70}$ Das zielt auf Verhandlungen über deutsche Identität, sagt aber weniger über den Nationalstaat als Akteur aus, dessen Bedeutungsverlust Doering-Manteuffel tatsächlich wohl etwas verfrüht, auf jeden Fall aber zu pau-

${ }^{67}$ Vgl. Wolfgang Streeck, Einleitung. Internationale Wirtschaft, nationale Demokratie?, in: Ders. (Hrsg.), Internationale Wirtschaft, nationale Demokratie. Herausforderungen für die Demokratietheorie, Frankfurt a. M./New York 1998, S. 11-58.

68 Ebenda, S. 13.

${ }^{69}$ Das bezeichnet allgemein eine zunehmende Bedeutung der Profitgenerierung durch Finanzgeschäfte gegenüber dem Handel und der Produktion von Gütern und Waren; vgl. Greta R. Krippner, Capitalizing on Crisis. The Political Origins of the Rise of Finance, Cambridge/ London 2011.

${ }^{70}$ Vgl. Hoeres, Gefangen, S. 433. 
schal verkündet. ${ }^{71}$ Abgesänge auf den schwachen, überforderten oder ineffizienten Staat waren seit der Regierbarkeitsdebatte der 1970er Jahre immer wieder zu hören, in den frühen 1990er Jahren war sogar von seiner „Aushöhlung“ die Rede. ${ }^{72}$ Diese Abgesänge haben sich jedoch als ebenso voreilig wie politisch motiviert erwiesen. Politikwissenschaftler warnten denn auch nachdrücklich davor, in der Forschung die Rede vom vermeintlichen Rückzug des Staats zu perpetuieren und dessen Transformation als Erosionsprozess zu beschreiben, da dies die Analyse des Wandels von Staatlichkeit bereits einseitig prädisponiere. ${ }^{73}$ Nationalstaaten waren und sind maßgebliche Akteure im Prozess der internationalen Liberalisierung des Handels, der Märkte und der Globalisierung. ${ }^{74}$ Das Ende der Ordnung von Bretton Woods Anfang der 1970er Jahre, in die die nationalen Ökonomien eingebunden waren, wurde durch Entscheidungen nationaler Regierungen besiegelt, die zunächst weiter versuchten, keynesianisch orientierte makroökonomische Koordination zu betreiben. Von Seiten der USA etwa folgte der Wandel von einer staats- zu einer marktzentrierten Währungsordnung durch die Lockerung von Kapitalkontrollen und Freigabe der Wechselkurse keinem marktliberalen grand design. Der Einfluss von Banken, unter denen unterschiedliche Vorstellungen über die Gestaltung des internationalen Währungssystems herrschten, blieb in den 1970er Jahren offensichtlich begrenzt, und die Entstehung eines transnationalen Finanzmarkts hatte in Gestalt des milliardenschweren Eurodollar-Markts bereits Ende der 1950er Jahre begonnen. ${ }^{75}$

Wenn die zeithistorische Forschung also nach dem Wandel von Handlungsfeldern und Handlungsmöglichkeiten des Nationalstaats fragt, sollte sie auch die Möglichkeit neuer Spielräume und die Erschließung neuer (Umwelt, Klima, Menschenrechte) beziehungsweise Ausweitung alter Regelungsfelder (insbesondere für die USA: innere und äußere Sicherheit sowie Kriminalität) berücksichtigen. Nationalstaaten delegierten eine Reihe von Regelungskompetenzen an internationale und europäische Instanzen oder Märkte. Dies ging einerseits mit einer Einschränkung nationalstaatlicher Handlungsautonomie einher, und Regelungsadressaten wie mobile Steuerzahler entzogen sich der staatlichen oder überstaatlichen Intervention. Andererseits aber entstanden im Rahmen der supranationalen Organisationen und Verhandlungssysteme neue Handlungs- und

71 „[D]as Zeitalter der Nationalstaaten ist vorbei“; Doering-Manteuffel, Deutsche Geschichte, S. 321.

${ }^{72}$ Vgl. R. A. W. Rhodes, The hollowing out of the state. The changing nature of public service in Britain, in: The Political Quarterly 65 (1994), S. 138-151.

${ }^{73}$ Vgl. Gunnar Folke Schuppert, Was ist und wie misst man den Wandel von Staatlichkeit?, in: Der Staat 47 (2008), S. 325-358. Für eine Kritik am britischen „decline of the state“-Narrativ vgl. Jon Pierre (Hrsg.), Debating Governance. Authority, Steering, and Democracy, Oxford 2000.

${ }^{74}$ Vgl. Lutz Raphael, Die Geschichte der Bundesrepublik schreiben als Globalisierungsgeschichte. Oder die Suche nach deutschen Plätzen in einer zusammenrückenden Welt seit 1949, in: Frank Bajohr u.a. (Hrsg.), Mehr als eine Erzählung. Zeitgeschichtliche Perspektiven auf die Bundesrepublik, Göttingen 2016, S. 203-218.

75 Vgl. Daniel J. Sargent, A Superpower Transformed. The Remaking of American Foreign Relations in the 1970s, Oxford 2015, hier S. 100-130. 
Einflussmöglichkeiten auf andere, schwächere Staaten; Entscheidungen, die im nationalen Kontext nicht durchsetzbar waren, konnten durch den Umweg über inter- oder transnationale Gremien realisiert werden. ${ }^{76}$ Welche Motive und Zielsetzungen, Interessen und Ideen, welche Handlungsspielräume gegenüber strukturellen Entwicklungen und welche Erwartungen die zeitgenössischen Akteure hatten - und wer überhaupt die Akteure waren -, ist in der historischen Forschung erst in Ansätzen untersucht. ${ }^{77}$

Darüber hinaus ist zu differenzieren, von wessen Handlungsmöglichkeiten eigentlich genau die Rede ist und mit welchen potenziellen Verschiebungen der Kräfteverhältnisse in den demokratischen Staaten wir es zu tun haben könnten: Möglicherweise zeichnete sich im Zuge der Europäisierung und Globalisierung eine Stärkung der nationalen Regierungen zu Lasten der parlamentarisch-demokratischen Entscheidungsfindung ab. Hier soll nun nicht die alte Rede vom Demokratiedefizit der Europäischen Union repliziert werden. Vielmehr geht es darum zu unterstreichen, dass es nicht nur den Wandel des National-, sondern auch des demokratischen Staats seit 1945 zu untersuchen gilt. Das ist nur möglich, wenn man nationale, transnationale und internationale Entwicklungen berücksichtigt, denn von allen Ebenen gingen gleichermaßen Veränderungsimpulse aus. Hierzu zählen auch Veränderungen im Staatsverständnis, Annahmen über die Rolle des Staats, Ideen also.

\section{Neoliberalismus: Ideengeschichte in nationaler, transnationaler und globaler Perspektive}

Generell ist es eine große Herausforderung für Arbeiten, die sich als Gesamtdarstellungen verstehen, nationale, transnationale und internationale Entwicklungen gemeinsam und in ihren Wechselwirkungen zu betrachten. Dies wird um so komplizierter, wenn man wie Doering-Manteuffel die Darstellung von der Ideengeschichte her konzipiert, jedoch nicht bei einer klassischen politischen Höhenkamm- oder Geistesgeschichte stehen bleibt, sondern die handlungsprägende Kraft, die gesellschaftliche und politische Wirkungsmacht von Ideen einbeziehen will. Ideen manifestieren sich auf der Ebene von Leitbegriffen und Diskursen und in Gestalt von Institutionen und Professionen; sie verdichten sich in Ideologien und Denksystemen, in Steuerungs- und Gestaltungsentwürfen, und sie werden verwirklicht durch die Implementation politischer Programme und Handlungs-

${ }^{76}$ Vgl. Renate Mayntz, Von der Steuerungstheorie zu Global Governance, in: Gunnar Folke Schuppert/Michael Zürn (Hrsg.), Governance in einer sich wandelnden Welt, Baden-Baden 2008, S. 43-60, hier S. 54 (= Sonderausgabe der Politischen Vierteljahresschrift, Bd. 41). Zur Ausweitung innenpolitischer Regelungsfelder vgl. Andreas Wirsching, Der Preis der Freiheit. Geschichte Europas in unserer Zeit, München 2012, S. 308-311.

77 Vgl. Daniel Sargent, The Cold War and the international political economy in the 1970s, in: Cold War History 13 (2013), S. 393-425. Jedoch gibt es eine Reihe historisch orientierter politikwissenschaftlicher Studien, die zur Erklärung von Gegenwartsentwicklungen bis in die 1940er Jahre zurückgehen; vgl. Jeffrey M. Chwieroth, Capital Ideas. The IMF and the Rise of Financial Liberalization, Princeton 2010. 
regulative. Als empirische Brücke zwischen Ideen und politischem Handeln mit realen gesellschaftlichen Konsequenzen bietet es sich an, wie in den Zeitbögen auch angedeutet, den Wandel von Leitbegriffen wie Fortschritt oder Freiheit im langen 20. Jahrhundert zu analysieren. Begriffe konfigurieren die soziale Welt, die vorgefundene genauso wie die erwünschte oder erwartete; in ihnen bündeln sich politische Interessen, Ideen und gesellschaftliche Ordnungsvorstellungen, und sie werden von bestimmten Akteuren propagiert, verbreitet und durchgesetzt. Ein semantischer Zugriff kann zum Beispiel zeigen, wie zeitgenössische Akteure versuchten, gesellschaftliche Entwicklungen sprachlich und theoretisch zu fassen, wie sich in ihren Wahrnehmungen ihre soziale Wirklichkeit konstituierte und wie sie aus diesen Wirklichkeitsdiagnosen wiederum Konsequenzen und Ziele für das politische Handeln ableiteten. ${ }^{78}$ In transnationaler Perspektive stellt sich hierbei mit der Übersetzbarkeit ein altes Problem der Begriffsgeschichte: Liberal ist nicht dasselbe wie liberal, Freiheit nicht dasselbe wie freedom oder liberty. Jedoch scheint in vielen modernen Industriegesellschaften seit den 1970er Jahren ein Bedeutungswandel politischer Schlüsselbegriffe stattgefunden zu haben, der mit einem zunehmenden Einfluss neoliberaler Denkströmungen und Bewegungen zusammenhing. ${ }^{79}$

Die Ideen- und Politikgeschichte des Neoliberalismus, den Doering-Manteuffel völlig zu Recht ins Zentrum des dritten Zeitbogens stellt, lässt sich denn auch nur in transnationaler Perspektive erhellen. Versteht man unter neoliberal die Weiterentwicklung des klassisch-liberalen Denkens in der Tradition Adam Smiths und des englischen New Liberalism (John Stuart Mill) seit den 1930er Jahren, ist zwischen mindestens vier ideellen Strömungen zu unterscheiden: zwischen dem deutschen Ordoliberalismus und den drei vor allem in den USA verbreiteten Spielarten der Public Choice-Schule um James Buchanan, des monetaristischen Strangs um Milton Friedman und des „evolutorischen Neoliberalismus“ von Hayek. ${ }^{80}$

Beim Neoliberalismus handelt es sich also keineswegs nur um einen „polemischen, heutzutage rein pejorativ gebrauchten " Begriff der Publizistik. ${ }^{81}$ Verengt man Neoliberalismus auf ein wirtschaftspolitisches Programm der Deregulierung und Privatisierung, wird es auch nicht gelingen, jene Neuordnung der Beziehungen zwischen Staat, Wirtschaft, Gesellschaft und Individuum zu erfassen, über die sich meines Erachtens der fundamentale Bruch zwischen der Epoche „nach dem Boom“ und den vorherigen Perioden beschreiben ließe. Hoeres will weder in der Bundesrepublik noch in der Europäischen Union und auch nicht in den USA die Durchsetzung einer (allein wirtschaftspolitisch definierten) „neoliberalen Agenda“ erkennen. Gegen Privatisierung und Deregulierung rechnet er

\footnotetext{
${ }^{78}$ Vgl. Ariane Leendertz/Wencke Meteling (Hrsg.), Die neue Wirklichkeit. Semantische Neuvermessungen und Politik seit den 1970er-Jahren, Frankfurt a. M./New York 2016.

${ }^{79}$ In transatlantischer Perspektive besonders relevant sind hierzu Daniel T. Rodgers, Age of Fracture, Cambridge 2011, und Eric Foner, The Story of American Freedom, New York 1998.

${ }^{80}$ Vgl. Thomas Biebricher, Neoliberalismus zur Einführung, 2. überarbeitete Aufl., Hamburg 2015.

${ }^{81}$ Dieses und folgendes Zitat bei Hoeres, Gefangen, S. $430 \mathrm{f}$.
} 
die Persistenz des Sozialstaats und die Zahl der Beschäftigten bei der Bundesagentur für Arbeit auf. Sozialstaatliche Strukturen und marktaffine Liberalisierungspolitik lassen sich allerdings nur schwer gegeneinander aufwiegen, zumal beide auf subtile Weise ineinandergreifen: Etwa, wenn der Kündigungsschutz gelockert wird oder betriebliche Sozialabgaben gesenkt werden, um die Flexibilität und Konkurrenzfähigkeit von Unternehmen im globalen Wettbewerb zu erhöhen. ${ }^{82}$ Gleichzeitig wandelten sich seit den 1990er Jahren politische Handlungslogiken, Zielsetzungen und Instrumente, beispielsweise durch die Implementation von Prinzipien des New Public Management in der Sozialverwaltung oder die Institutionalisierung von Quasi-Märkten und Wettbewerbsbedingungen in der Hochschulfinanzierung, mit denen sich zugleich Einstellungen und Selbstverständnisse der Akteure veränderten. Die sozialwissenschaftliche Forschung spricht hier, allerdings oft mit stark normativem Einschlag, von einer "Ökonomisierung“ politischer und gesellschaftlicher Handlungszusammenhänge, die durch Begriffe und Managementinstrumente aus der Unternehmenswelt und durch die Erzeugung von Wettbewerbs- und Marktbeziehungen umgestaltet wurden. ${ }^{83}$

Dass es sich bei den Hartz-Reformen um ein Programm der Verhaltenssteuerung und Subjektivierung im Sinne der Paradigmen der Aktivierung und Responsibilisierung des Individuums handelte, die länderübergreifend als Charakteristika neoliberaler Gouvernementalität beschrieben werden, ${ }^{84}$ verbleibt in Hoeres' Perspektive somit im toten Winkel. Und dass es Ronald Reagan entgegen seiner Ankündigungen nicht gelang, die Staatsquote zu senken und die Sozialversicherung abzuschaffen, lag weniger an seinem sozialen Gewissen als an der Ausweitung des Verteidigungsetats, dem Widerstand des Kongresses und an wahltaktischen Überlegungen. Bedeutende Weichenstellungen der Regierung Reagan bildeten Steuersenkungen, die die Staatsverschuldung in Rekordhöhe trieben, die unternehmensfreundliche Schwächung zahlloser Aufsichts- und Regulierungsbehörden und die Deregulierung der Versicherungs- und Finanzwirtschaft,

${ }^{82}$ Vgl. Wencke Meteling, Nationale Standortsemantiken seit den 1970er-Jahren, in: Leendertz/ Meteling (Hrsg.), Neue Wirklichkeit, S. 207-241.

${ }^{83}$ Vgl. Gary S. Schaal/Matthias Lemke/Claudia Ritzi (Hrsg.), Die Ökonomisierung der Politik in Deutschland. Eine vergleichende Politikfeldanalyse, Wiesbaden 2014, und Rainer DiazBone/Gertraude Krell (Hrsg.), Diskurs und Ökonomie. Diskursanalytische Perspektiven auf Märkte und Organisationen, Wiesbaden 2009. Für eine begrifflich-analytische Differenzierung vgl. Matthias Kettner, Ein Vorschlag zur Unterscheidung von Ökonomisierung und Kommerzialisierung, in: Ders./Peter Koslowski (Hrsg.), Ökonomisierung und Kommerzialisierung der Gesellschaft. Wirtschaftsphilosophische Unterscheidungen, München 2011, S. 3-21.

${ }^{84}$ Vgl. Peter Miller/Nikolas Rose, Governing the Present. Administering Economic, Social and Personal Life, Cambridge/Malden 2008; Ulrich Bröckling/Susanne Krasmann/Thomas Lemke (Hrsg.), Gouvernementalität der Gegenwart. Studien zur Ökonomisierung des Sozialen, Frankfurt a. M. 2000; Ulrich Bröckling, Das unternehmerische Selbst. Soziologie einer Subjektivierungsform, Frankfurt a.M. 2007; Stephan Lessenich, Die Neuerfindung des Sozialen. Der Sozialstaat im flexiblen Kapitalismus, Bielefeld 2008, und Wendy Brown, Undoing the Demos. Neoliberalism's Stealth Revolution, New York 2015. 
die der Demokrat Bill Clinton in den 1990er Jahren nahtlos fortsetzte. ${ }^{85}$ Bezüglich der Europäischen Union unterstreichen Politikwissenschaftler die Rolle des europäischen Wettbewerbsrechts als Hebel marktorientierter Liberalisierung bereits seit den 1970er Jahren. Der Europäische Gerichtshof und die Europäische Kommission konnten seitdem rechtliche Schritte gegen nationale Handels- und Mobilitätsbeschränkungen einleiten, die den freien Wettbewerb privater Dienstleister auf dem Markt beeinträchtigten. Auf dieser rechtlichen Basis konnten letztere grundsätzlich Wettbewerbsgleichheit mit öffentlichen Anbietern und Einrichtungen einfordern. ${ }^{86}$

Mit dem Neoliberalismus verbindet sich wesentlich mehr als ökonomische Theorie und wirtschaftspolitische Programmatik, wenn man die demokratie- und gesellschaftstheoretischen Implikationen der dem neoliberalen Denkzusammenhang zuzuschlagenden Public Choice- und Rational Choice-Theorie betrachtet. In diesen Zusammenhang wäre auch die Humankapitaltheorie einzuordnen, die mit dem Namen des Ökonomen Gary Becker verbunden ist. ${ }^{87}$ Wie die Politikwissenschaftlerin Sonia Amadae zeigt, entwarfen die Vordenker der Rational Choice- und Public Choice-Theorie um Kenneth Arrow, William H. Riker, Gordon Tullock, Mancur Olson und Buchanan seit den 1950er Jahren eine neue Demokratietheorie, die um den rationalen, eigennützigen und strategischen Akteur zentriert war. ${ }^{88}$ Arrow zufolge war es logisch unmöglich, aus der Summe individueller Präferenzen das Allgemeinwohl zu ermitteln und rationale Gruppenentscheidungen

${ }^{85}$ Vgl. Mark Blyth, Great Transformations. Economic Ideas and Institutional Change in the Twentieth Century, Cambridge/New York 2002, S. 180-201. Für eine abgewogene Bilanz der Präsidentschaft Reagans vgl. Sean Wilentz, The Age of Reagan. A History 1974-2008, New York 2008, hier S. 273-287. Zum nicht nur sprichwörtlichen Ende von „welfare as we know it“ durch den „Personal Responsibility and Work Opportunity Reconciliation Act“ von 1996 vgl. Alice O'Connor, Poverty Knowledge. Social Science, Social Policy, and the Poor in Twentieth Century U.S. History, Princeton/Oxford 2001, hier S. 284-291. Es war der Demokrat Clinton, der 1996 in seiner Rede zur Lage der Nation verkündete, „the era of big government is over".

${ }^{86}$ Vgl. Fritz Scharpf, Regieren in Europa. Effektiv und demokratisch?, Frankfurt a. M. 1999, hier S. 57-69; Martin Höpner, Wie der Europäische Gerichtshof und die Kommission Liberalisierung durchsetzen. Befunde aus der MPIfG-Forschungsgruppe zur Politischen Ökonomie der europäischen Integration, Köln 2014; URL: http:/ / www.mpifg.de/pu/mpifg_dp/dp14-8. pdf [10.1.2017]. Unter dem Schlagwort „New Constitutionalism“ untersuchen Rechts- und Politikwissenschaft seit einigen Jahren, in explizit neoliberalismuskritischer Perspektive, inwiefern sich seit dem Ende des Kalten Kriegs über die internationale Rechtsprechung und Rechtsetzung sowie transnationale, halb- oder nichtstaatliche Regelungsstrukturen de facto eine neue, wirtschaftsfreundliche Rechtsordnung etabliert, die demokratische Aushandlungen und den nationalen Rechtsstaat unterminiert; vgl. Stephen Gill/A. Claire Cutler (Hrsg.), New Constitutionalism and World Order, Cambridge 2014.

${ }^{87}$ Das komplizierte Ideenkonglomerat schlüsselt Rodgers, Age of Fracture, hier S. 41-76, auf, aus dem Ende der 1970er Jahre der Markt als Metapher für die Gesellschaft entstand, während er in der klassischen liberalen Theorie den Ort wirtschaftlicher Austauschbeziehungen darstellte, der neben den Sphären des Politischen und des Privaten existierte.

${ }^{88}$ Vgl. S. M. Amadae, Rationalizing Capitalist Democracy. The Cold War Origins of Rational Choice Liberalism, Chicago 2003, die damit Zusammenhänge zwischen „Cold War Rationality" und neoliberalem Denken herausstellt. 
zu treffen. Ohne Zwang seien rationale, nutzenkalkulierende Individuen - und andere gab es der radikal rationalistisch-individualistischen Theorie zufolge nicht - nicht in der Lage, im gemeinsamen Interesse zu handeln, so Olson. Die Demokratie konstituierte sich in dieser Sicht durch freiwillige Austausch- und Vertragsbeziehungen rationaler Individuen, die allein der Souveränität des Markts unterworfen waren. In der Konsequenz resultierte hieraus eine Umdeutung zentraler Begriffe der Demokratie, wie die Politikwissenschaftlerin Wendy Brown anhand der Rechtsprechung des Obersten Gerichtshofs der USA im 21. Jahrhundert zeigt. So hob der Supreme Court 2010 im Urteil Citizens United v. Federal Election Commission mit Bezug auf die Redefreiheit den Unterschied zwischen natürlichen Personen und Unternehmen auf. Limitiere man die Wahlkampfspenden von Unternehmen, würden diese auf dem politischen Marktplatz der Ideen in ihrer Redefreiheit gehindert; schränke man ihre Redefreiheit ein, verwehre man damit den Bürgern und somit anderen Marktakteuren den Zugang zu Informationen. Das Grundrecht der freien Rede mutierte damit zur Information für rationale Akteure auf einem Markt von Ideen, Meinungen und Wählerstimmen. Das habe schwerwiegende Folgen für die Demokratie, so Brown: Die Kaufkraft der Unternehmen, die sich teure Kampagnen leisten können, verdränge andere Stimmen; die Ausübung der Redefreiheit werde zu einer Geldfrage, und die Schwächeren, deren politische Rechte der demokratische Staat eigentlich schützen müsse, würden im Wettbewerb verdrängt. Brown spricht von einer neoliberalen Rationalität des Regierens, als deren Kern ihr eine Ökonomisierung aller Bereiche gesellschaftlichen Handelns gilt. ${ }^{89}$

Hier wird eine wesentlich tiefergehende Transformation der (amerikanischen) Gesellschaft und Demokratie erkennbar, als sie in weiten Teilen der Geschichtswissenschaft bislang empirisch bewusst ist. Browns Gegenwartsanalyse fehlt es indes an historischer Tiefe und es wird nicht klar, wie genau der Wandel der Semantiken und des Denkhaushalts bis in die Urteilsbegründung eines Obersten Richters der Vereinigten Staaten vordringen konnten. Allmählich kristallisieren sich in historischen Arbeiten jedoch immer mehr die Allianzen zwischen Ideengebern und Interessengruppen heraus, werden konzertierte Strategien des Ideenmarketings und der Popularisierung, die Lobbyaktivitäten von Firmen und Unternehmen und schließlich eine Neuordnung von Machtverhältnissen in der politischen Ökonomie der USA namentlich seit den 1970er Jahren sichtbar. ${ }^{90}$ Anhand des bislang wenig beachteten Netzwerks der Virginia School um James Buchanan lässt sich so auch zeigen, dass diese Strategien nicht einfach auf einen

\footnotetext{
${ }^{89}$ Vgl. Brown, Undoing the Demos, hier S. 151-173.

${ }^{90}$ Vgl. Angus Burgin, The Great Persuasion. Reinventing Free Markets since the Depression, Cambridge/London 2012; Jennifer Burns, Goddess of the Market. Ayn Rand and the American Right, New York 2009; Kim Phillips-Fein, Invisible Hands. The Making of the Conservative Movement from the New Deal to Reagan, New York 2009, und Benjamin C. Waterhouse, Lobbying America. The Politics of Business from Nixon to NAFTA, Princeton 2014.
} 
Rückbau des Staats zielten, sondern vielmehr auf dessen feindliche Übernahme: eine andere Art des Marsches durch die Institutionen. ${ }^{91}$

Verstehen wir die USA als neoliberales Gemeinwesen, können sie uns heuristisch als tertium comparationis dienen, vor dessen Hintergrund sich deutsche Entwicklungen mit der ideengeschichtlich als neoliberal definierbaren Wirtschaftsund Demokratietheorie und gouvernementalen Logiken in Beziehung setzen lassen. Unter neoliberal ist dann weniger ein kohärentes (wirtschafts-)politisches Programm intentional handelnder Akteure zu begreifen, sondern es ist damit eine Neuorganisation der Beziehungen zwischen Staat, Wirtschaft, Gesellschaft und Individuum umschrieben, die sich im diachronen Vergleich zur Periode des Golden Age, des eingebetteten Kapitalismus der Jahrzehnte seit 1930 und der Ära des Kalten Kriegs sowie der Periode der Hochindustrialisierung und internationalen Verflechtung vor dem Ersten Weltkrieg konturieren lässt. Die gegenwartsnahe Zeitgeschichte eröffnet somit auch neue Perspektiven auf die Geschichte des langen 20. Jahrhunderts. In zeitgeschichtlichen Arbeiten hat die Einheit des kurzen 20. Jahrhunderts und der „moralischen“ Epoche der Weltkriege, Gewaltherrschaft und Ideologien ihre zeitweilig dominierende Stellung verloren, und der Eindruck gegenwärtiger Entwicklungen generiert neue Fragestellungen, Kategorien und Themenkomplexe für die historische Forschung. So schreiben wir die Geschichte laufend neu, entdecken neue Narrative, Strukturierungs- und Darstellungsmöglichkeiten und gewinnen neue Erkenntnisse über die Zeitgeschichte, die mit unseren Lebensgeschichten und Erfahrungsräumen ebenso verwoben ist wie mit der Gegenwart, aus der wir zurückblicken.

${ }^{91}$ Vgl. Nancy MacLean, Chaining Leviathan. The Decades-Long Plan of the Radical Right to Shackle Democracy, New York 2017 (i.E.). 
Bereitgestellt von | Max Planck eBooks

Angemeldet

Heruntergeladen am | 03.04.18 11:56 